\title{
Nephrogenic systemic fibrosis associated with stromal and vascular calcification, report of two cases
}

Nephrogenic systemic fibrosis (NSF) is a systemic fibrosing disorder characterized by the development of large indurated plaques on the skin, primarily in patients with end-stage renal disease (ESRD). Skin biopsy reveals an increased frequency of $\mathrm{CD}_{3} 4^{+}$and Factor XIIa+ cells. Microscopic calcification has been reported in the skin biopsies of rare cases of NSF. The etiology and significance of this calcification is not clear. We present two cases of typical NSF, for which marked stromal and vascular calcification was identified on skin biopsy. In both cases, the calcification was within typical NSF lesions and was intimately associated with aggregates of CD34 ${ }^{+}$, Factor XIIIa+ spindle and stellate cells. This particular pattern of calcification strongly argues against either nonspecific dystrophic calcification or coincidental metastatic calcification. These findings suggest that calcification may be intrinsic to the pathophysiology of NSF, at least in a subset of NSF patients. In addition, the vascular calcification involving small arteries and arterioles in these two cases morphologically resembled calciphylaxis, which is another poorly understood complication of ESRD. This resemblance raises the possibility that NSF may predispose to or develop in the vicinity of indolent lesions of early-stage calciphylaxis. We propose that skin biopsies performed as part of a diagnostic workup for suspected NSF should preferably include the deep dermis and subcutis, in order to assess possible vascular calcification.

Song J, Volkov S, Shea CR, Alegre M-L, Salgia R, Gregg K, Curran JJ, Woodruff J, Krausz T, Levine JS, Sweiss NJ. Nephrogenic systemic fibrosis associated with stromal and vascular calcification, report of two cases.

\section{Jie Song', Suncica Volkov², Christopher R. Shea ${ }^{3}$, Maria-Luisa Alegre ${ }^{4}$, Reena Salgia ${ }^{5}$, Kevin Gregg ${ }^{4}$, James J. Curran ${ }^{4}$, James Woodruff ${ }^{4}$, Thomas Krausz ${ }^{1}$, Jerrold S. Levine $^{6}$ and Nadera J. Sweiss ${ }^{7}$}

${ }^{1}$ Department of Pathology, University of Chicago, Chicago, IL, USA,

${ }^{2}$ Section of Rheumatology, Department of Medicine, University of IL, IL, USA, ${ }^{3}$ Section of Dermatology, Department of Medicine, University of Chicago,

${ }^{4}$ Department of Medicine, University of Chicago, Chicago, IL, USA,

${ }^{5}$ Department of Medicine, University of Michigan, Ann Arbor, Ml, USA,

${ }^{6}$ Section of Nephrology, Department of Medicine, University of IL, IL, USA, and

${ }^{7}$ Section of Rheumatology, Department of Medicine, University of Chicago, Chicago, IL, USA

Nadera J. Sweiss, MD, Section of Rheumatology, Department of Medicine, University of Chicago, 5841 S Maryland Avenue, MC 0930, Chicago, IL 60637, USA

Tel: (773) 702-5379

Fax: (773) 834-7065

e-mail: nsweiss@medicine.bsd.uchicago.edu

Accepted for publication October 16, 2008

J Cutan Pathol 2009; 36 (Suppl. 1): 31-34. (C) 2008 Blackwell Munksgaard.

Nephrogenic systemic fibrosis (NSF), also known as nephrogenic fibrosing dermopathy, is a systemic fibrosing disorder primarily affecting patients with end-stage renal disease (ESRD). Patients develop progressive, often disabling, indurated plaques and confluent papules on their skin. ${ }^{1}$ Although NSF has been linked to gadolinium, its etiology is not fully established. Calciphylaxis is another poorly understood complication of ESRD, characterized by calcification of small dermal and subcutaneous arteries, skin ulceration and necrosis. Because both NSF and calciphylaxis are rapidly progressive skin conditions with potentially catastrophic outcomes, careful differential diagnosis and timely treatment are important. $^{2}$

Microscopic calcifications are occasionally seen in the skin biopsies of patients with NSF. The etiology and significance of this finding remain under debate. Some authors think that it is a non-specific dystrophic calcification, occurring in older lesions, and is not intrinsic to $\mathrm{NSF}^{3}$ while others suggest that it occurs in only a subset of NSF patients and indicates a poorer prognosis. ${ }^{4}$ Moreover, as NSF patients often have impaired calcium metabolism because of accompanying ESRD, any calcific lesion must raise the possibility of coincidental metastatic calcification. 


\section{Song et al.}

Here, we present two cases of NSF, for which the typical skin lesions were intimately associated with marked stromal and vascular calcification. This particular distribution pattern makes it unlikely that the calcific lesions can be attributed to either non-specific dystrophic calcification or metastatic calcification. Our cases raise the possibility that, in certain patients, calcification may contribute to the pathophysiology of NSF. Alternatively, as hinted by a recent study, pre-existing calcium deposition may facilitate the toxicity of gadolinium and predispose to the development of NSF. ${ }^{5}$ In view of this potential etiologic association as well as the significant morbidity associated with vascular calcification, a deep skin biopsy, including the deep dermis and subcutis, may be preferred as part of the diagnostic evaluation in patients suspected of having NSF.

\section{Case 1}

A 65-year-old African American woman, with a history of hypertension, diabetes mellitus and ESRD, had been maintained on hemodialysis for 8 years. Her course was notable for repeated thrombotic events, involving upper extremity catheters and the infrarenal inferior vena cava. Her recurrent thrombosis had been attributed to hyperhomocysteinemia (30.0 $\mu \mathrm{mol} / \mathrm{l}$, normal range 4.5-13.0) and elevated IgA anti-beta-2 glycoprotein I (anti- $\beta 2 \mathrm{GPI}$ ) antibodies (35.8 and 22.6 standard international units $[\mathrm{SAU}] / \mathrm{ml}$, performed twice, 8 weeks apart, normal $<20$ ). She presented with thrombosis of her translumbar dialysis catheter, despite adequate anticoagulation, and pain in both her arms. Physical examination was notable for multiple areas of mildly hyperpigmented, thickened, indurated skin, located symmetrically on her hands, mid to upper arms, ankles and mid-abdomen. Her face and upper trunk were spared. She had limited range of motion about her elbows, wrists and knees. Antinuclear antibody, anti-topoisomerase antibody (antiScl-70), anti- $\beta 2$ GPI antibodies and anti-cardiolipin antibodies were not detected during this admission. There was no known gadolinium exposure prior to the onset of her skin disease. Since beginning dialysis, her serum calcium had always been within the normal range, whereas her inorganic phosphate had been mostly elevated, ranging from 4.2 to $9.0 \mathrm{mg} / \mathrm{dl}$.

A skin biopsy of a lower leg lesion showed prominent dermal and subcutaneous fibrosis, increased CD $34^{+}$, Factor XIIIa + spindle and dendritic cells and increased mucin deposition (colloidal iron stain), characteristic of NSF. In addition, there was marked dermal stromal calcification as well as deep dermal and subcutaneous vascular calcification (von Kossa stain), resembling calciphylaxis. The patient subsequently developed multiple comorbidities, including severe hypotension, ischemic necrosis of the right hand and rapid declination of mental status. Death occurred 1 month after presentation.

\section{Case 2}

A 39-year-old Caucasian woman, with a history of type I diabetes mellitus and ESRD, had been maintained on hemodialysis for 7 years, following renal allograft failure from a kidney-pancreas transplant in 1999. She initially noticed some mild swelling of lower legs in September 2005. In February 2006, she was hospitalized for intestinal bleeding, which was caused by a pancreatic-intestinal fistula. Three weeks later, she developed progressive thickening and induration of the skin on her feet, lower legs, upper arms, hands and lower back (Fig. 1). These caused severe limitation in the range of motion about her
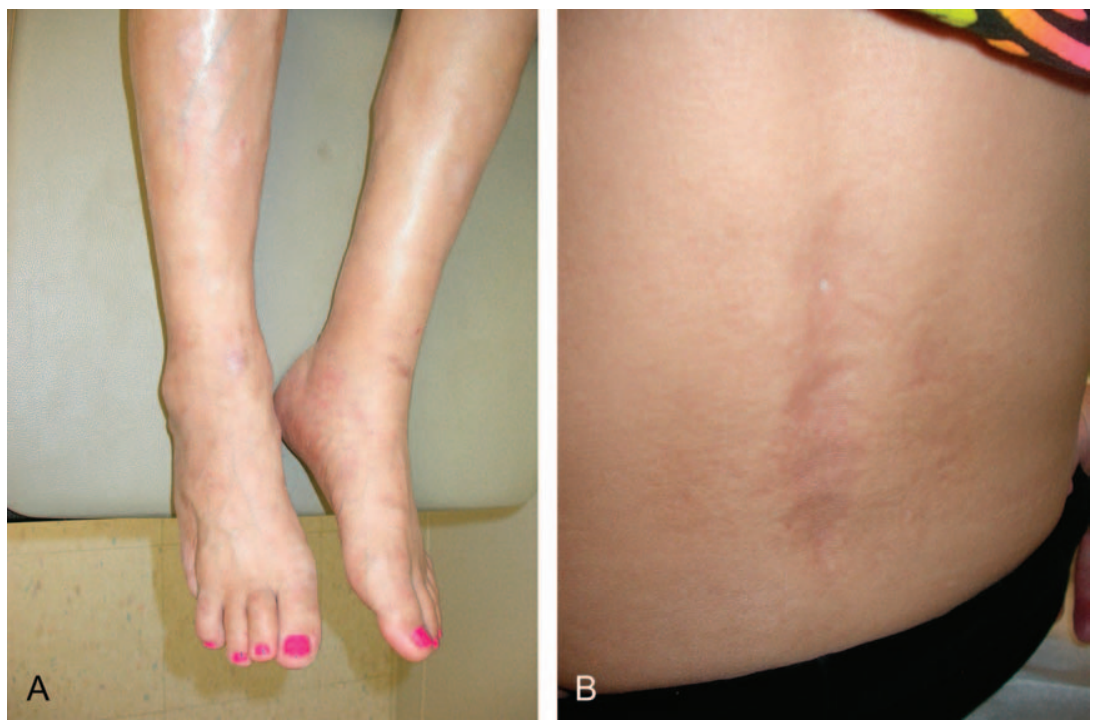

Fig. 1. A) Lower leg skin lesions. B) Lower back skin lesions. 


\section{Calcification and NSF}

knees and ankles, and she eventually became wheelchair bound. At the same time, firm nodules developed on her palms, interfering with her hand function. There was no known gadolinium exposure prior to the onset of her skin disease. Over the preceding year, her serum calcium had been mostly normal, ranging from 9.4 to $11.0 \mathrm{mg} / \mathrm{dl}$, whereas her inorganic phosphate was slightly elevated to $5.0 \mathrm{mg} / \mathrm{dl}$.

Skin biopsy of a lower leg lesion showed thick collagen bundles, increased stromal mucin (colloidal iron stain) and increased spindle and stellate cells that were positive for CD34 and Factor XIIIa. A diagnosis of NSF was made. She was started on mycophenolate mofetil. Excision of palmar nodules was also performed and too revealed characteristic NSF histopathology, with increased CD $34^{+}$and Factor XIIIa + cells. Stromal calcification, as well as vascular calcification involving small deep dermal and subcutaneous arteries, was present in all specimens (Fig. 2). Calcification was intimately associated with aggregates of spindle and stellate cells. Stromal calcification was particularly prominent in the palmar nodules and resembled calcinosis. The patient was treated with mycophenolate mofetil, intravenous immunoglobulin (IVIG) and rituximab for her disabling skin disease. The skin stiffness and thickening improved markedly. The patient is currently alive and well.

\section{Discussion}

Cowper et al. ${ }^{1}$ first described NSF in 2000 as a scleromyxedema-like disease in 15 dialysis patients, who developed diffuse thickening and induration of their skin, with histology showing increased fibroblast-like dermal cells associated with collagen remodeling and mucin deposition. Although the etiology of NSF is still unclear, an association with gadolinium, a component of magnetic resonance imaging (MRI) contrast agents, has been suggested by multiple studies. ${ }^{6}$ However, both of our cases lacked a clear history of gadolinium exposure. Currently, there is no established treatment for NSF.

Calcification is an uncommon finding in NSF biopsies. Although it has been observed in several cases, there is no consensus on its etiology or significance. Cowper et al. ${ }^{3}$ suggested that such calcification may be non-specific and dystrophic in nature. In contrast, Edsall et al. ${ }^{4}$ reported two cases of NSF with extensive stromal calcium deposition, which they believed was a manifestation of metastatic calcification. The biopsy of one patient also showed calciphylaxis-like vascular calcification. They also suggested that both calcification and fibrosis may be related to the activity of transforming growth factor$\beta /$ Smad signaling pathway. Ting et al. ${ }^{7}$ reported the autopsy of a NSF patient that revealed extensive fibrosis and stromal calcification in the dermis and skeletal muscle, as well as vascular calcification in multiple internal organs. Hershko et al. reported two cases of NSF with osteoclast-like giant cells and suggested that these cases may represent a variant of NSF. One of these patients had stromal and vascular calcification morphologically similar to our cases. ${ }^{8}$ Swartz et al. ${ }^{9}$ noted focal dermal calcification in 1 of 13 NSF patients, but further details were not
Fig. 2. A) Biopsy of palmar lesion shows increased fibroblast-like cells and marked stromal calcification $(\times 25)$. B) Small subcutaneous arteries with marked medial calcification $(\times 100)$. Inset shows von Kossa stain. C) CD34 immunohistochemical stain (×400). D) Factor XIIIa immunohistochemical stain $(\times 400)$.
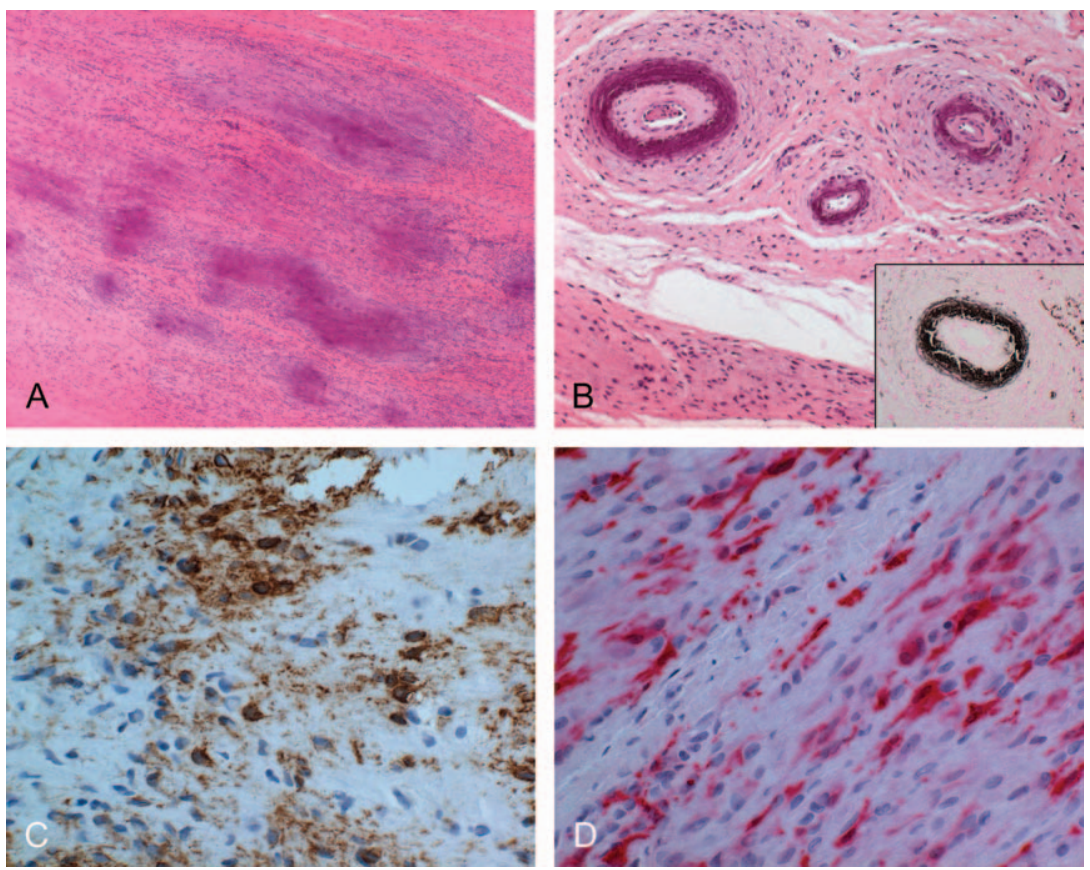


\section{Song et al.}

published. Markus et al. ${ }^{10}$ reported subcutaneous stromal calcification in two of four NSF patients. Finally, Lewis et al. ${ }^{11}$ reported one case with clinically and histologically overlapping NSF and calciphylaxis.

Our cases may provide insight into the role of calcification in NSF. In both cases, calcification was intimately associated with aggregates of $\mathrm{CD} 34^{+}$and Factor XIIIa + spindle and stellate cells, often tightly surrounded by these cells. It should be noted that calcified lesions are not uncommon in dialysis patients as a result of metastatic calcification. However, the anatomic proximity observed in our cases argues against the possibility of coincidental metastatic calcification. Non-specific dystrophic calcification seems equally unlikely because it is supposed to be limited to the stroma, and both of our patients had vascular calcifications. We hypothesize that in certain patients with NSF, calcification may be intrinsic to the disease process.

A recent report by Boyd et al. suggests a pathophysiologic mechanism for this association. In their report, gadolinium was detected in dermal vessel walls, but only in areas with concomitant calcium phosphate deposition. ${ }^{5}$ Because gadolinium is toxic only in its free form, whereas gadolinium-based MRI contrast agents contain exclusively chelated gadolinium, toxicity requires that free gadolinium somehow be released into tissues. Calcium phosphate particles may provide a mechanism for the release of free gadolinium by a transmetallation reaction, and thereby serve as a precipitating factor for NSF.

There may be an additional significance to the vascular calcification seen in our two cases. Calcification of subcutaneous blood vessels, including small arteries and arterioles, is the pathological hallmark of calciphylaxis. Such vascular calcification may be accompanied by intimal hyperplasia and thrombosis, leading to ischemia, skin ulceration and necrosis. ${ }^{12}$ The morphological resemblance to calciphylaxis in our two cases raises the possibility that NSF may predispose to or harbor indolent lesions characteristic of an early stage of calciphylaxis. Cases with coexisting NSF and calciphylaxis have been reported in the literature. ${ }^{11}$ In view of this potential association, a deep skin biopsy reaching the subcutis may be indicated in patients with suspected NSF.

In summary, we present two cases of NSF with concomitant stromal and vascular calcification. We hypothesize that the association of calcification with NSF, rather than being a coincidence, may offer insight into the pathophysiology of this puzzling syndrome. Future studies should examine the validity of our observation because a greater understanding of the role of calcification in NSF may lead to new strategies for both treatment and prevention.

\section{References}

1. Cowper SE, Robin H, Steinberg S. Scleromyxedema-like cutaneous disease in renal dialysis patients. Lancet 2000; 356: 1000.

2. Weenig RH, Gibson LE, el-Azhary R. The role of the hospital dermatologist in the diagnosis and treatment of calciphylaxis and nephrogenic systemic fibrosis. Semin Cutan Med Surg 2007; 26: 163.

3. Cowper SE, Su LD, Bhawan J, Robin HS, LeBoit PE. Nephrogenic fibrosing dermopathy. Am J Dermatopathol 2001; 23: 383.

4. Edsall LC, English JC, Teague MW, Patterson JW. Calciphylaxis and metastatic calcification associated with nephrogenic fibrosing dermopathy. J Cutan Pathol 2004; 31: 247.

5. Boyd AS, Zic JA, Abraham JL. Gadolinium deposition in nephrogenic fibrosing dermopathy. J Am Acad Dermatol 2007; 56: 27.

6. Othersen JB, Maize JC, Woolson RF, Budisavljevic MN. Nephrogenic systemic fibrosis after exposure to gadolinium in patients with renal failure. Nephrol Dial Transplant 2007; 22: 3179.

7. Ting WW, Stone SM, Madison KC, Kurtz K. Nephrogenic fibrosing dermopathy with systemic involvement. Arch Dermatol 2003; 139: 903.

8. Hershko K, Hull C, Ettefagh L, et al. A variant of nephrogenic fibrosing dermopathy with osteoclast-like giant cells: a syndrome of deregulated matrix remodeling. J Cutan Pathol 2004; 31: 262.

9. Swartz RD, Crofford LJ, Phan SH, Ike RW, Su LD. Nephrogenic fibrosing dermopathy: a novel cutaneous fibrosing disorder in patients with renal failure. Am J Med 2003; 114: 563.

10. Markus JS, James AJ, Nunez-Gussman JK, Sheehan AM, Fegan L, Hsu S. Nephrogenic fibrosing dermopathy. J Am Acad Dermatol 2005; 52: 166.

11. Lewis KG, Lester BW, Pan TD, Robinson-Boston L. Nephrogenic fibrosing dermopathy and calciphylaxis with pseudoxanthoma elasticum-like changes. J Cutan Pathol 2006; 33: 695.

12. Arseculeratne G, Evans AT, Morley SM. Calciphylaxis, a topical overview. J Eur Acad Dermatol Venereol 2006; 20: 493.

\section{Note added after online publication: conflicts of interest}

None declared. 